\title{
THE BUST OF FRANCESCO II GONZAGA: FROM DIGITAL DOCUMENTATION TO 3D PRINTING
}

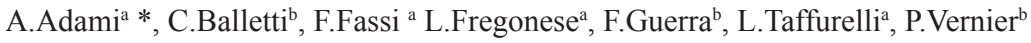 \\ a Politecnico of Milan, 3D Survey Group, p.za L.da Vinci 32, Milan, Italy - (andrea.adami, francesco.fassi, luigi.fregonese, \\ laura.taffurelli)@polimi.it \\ ${ }^{\mathrm{b}}$ Università Iuav of Venezia, Lab Systems, Photogrammetry Lab Santa Croce 191, Venice, Italy, (caterina.balletti, guerra2, paolo. \\ vernier)@iuav.it
}

\section{Commission VI, WG VI/4}

KEY WORDS: Cultural Heritage documentation, statues digitalization, triangulation range sensors, photogrammetry, dense image matching, modelling, 3D printing.

\begin{abstract}
:
Geomatics technics and methods are now able to provide a great contribution to the Cultural Heritage $(\mathrm{CH})$ processes, being adaptable to different purposes: management, diagnosis, restoration, protection, study and research, communication, formation and fruition of the Cultural Heritage. This experimentation was done with an eye to encouraging and promoting the development of principles and good practices for recording, documentation and information management of cultural heritage

This research focuses on the documentation path of a cultural asset, in particular a Renaissance statue, aimed to achieve a three dimensional model useful for many digital applications and for solid reproduction. The digital copy can be used in many contexts and represents an efficient tool to preserve and promote $\mathrm{CH}$. It can be included in virtual museum archives and catalogues, shared on network with cultural operators and users, and it permits the contextualization of the asset in its artistic and historical background. Moreover, the possibility to obtain a hard copy, reproduced through 3D printing, allows to reach new opportunities of interaction with $\mathrm{CH}$.

In this article, two techniques for the digitization of the terracotta bust of Francesco II Gonzaga, in the City Museum of Mantua, are described: the triangulation scanner and dense image matching photogrammetry. As well as the description of the acquisition and the elaborations, other aspects are taken into account: the characteristics of the object, the place for the acquisition, the ultimate goal and the economic availability. There are also highlighted the optimization pipeline to get the correct three-dimensional models and a 3D printed copy. A separate section discusses the comparison of the realized model to identify the positive and negative aspects of each adopted application.
\end{abstract}

\section{INTRODUCTION}

\subsection{The documentation of Cultural Heritage assets}

The documentation of Cultural Heritage $(\mathrm{CH})$ is a research field that recently has had a very important growth. This is due in part to technological developments that led to new methods and instruments for the digital acquisition of cultural assets. Among these, range based systems have surely a very important role and widespread as they allow to acquire precise data in real time. In addition, the intersection between photogrammetry and computer vision led to new algorithms and software enabling the digital reconstruction of an object using photographs in a semi automatic way (Fassi et al, 2013).

The remarkable development in the field of digitization of $\mathrm{CH}$ is also connected with a greater awareness of the crucial role of cultural assets both as an economic resource and as a cultural element of recognition and sharing. Having ascertained this role of $\mathrm{CH}$, the aim of the documentation phase is not only the recording of all available information through the construction of a three-dimensional model, but it includes a number of related activities: the implementation of digital archives, the cataloguing and the network sharing of models are only the first step in the enhancement of $\mathrm{CH}$.

The ability to obtain accurate and very similar to the originals three-dimensional models allows also other very interesting cultural operations. First of all the contextualization of the asset: it often happens that objects are extracted from their original context and are preserved in the museum rooms. In those cases it is not possible to really understand the relationship of the object with its native context and the experience for the visitor is impoverished. The re-contextualization, even just virtual (Adami et al, 2012, Taffurelli et al, 2011), enables to fully grasp the characteristics of the object.

The three-dimensional digital model can also be the starting point for new forms of economy in the field of $\mathrm{CH}$ : new marketing for museums and possibility of tourist souvenirs.

The ability to make three-dimensional solid prints of architectures or architectural elements allow the visitor and user of museums to have new perceptions. It happened in the case of the model of the Codussi facade of the church of San Michele in Isola, reproduced through 3D printing for the exhibition on 'Camaldolesian order at the Museo Correr in Venice (Balletti et al, 2012).

All these observations occurred during the digitization of the bust of Francesco II Gonzaga, in the City Museum of Mantua. To obtain the digital model of the terracotta bust various methods and instruments were employed, to verify not only the most suitable technique, but also to highlight for each method the positive and negative aspects. In particular the comparison regards triangulation scanners (Range 7 and Vivid 9i) and the 
recent photogrammetric software using dense image matching algorithms.

To demonstrate that a digital copy can be used in many contests and it can be considered a starting point for further applications, we tested the solid print. The $3 \mathrm{D}$ digital model was printed in different scales to check if the quality of the model was sufficient. Moreover the model printed at the reduced scale 1:5 demonstrates the feasibility of a new market that can be linked to cultural heritage (Neumuller et al, 2014), instead the detail printed in scale $1: 1$ shows the achievable richest detail and that the model can be used in 1:1 reproduction for museums or temporary exhibitions. In this article, after an historical-artistic introduction on the terracotta bust, there is a description of the methods and tools used in the digitization stage. In particular, we will describe the phase of data acquisition, processing and optimization to get the three-dimensional models and a printed copy. A separate section will discuss the comparison of the models realised by two different techniques, triangulation system and digital photogrammetry, to identify the positive and negative aspects of each application.

\subsection{The terracotta bust of Francesco II Gonzaga}

The bust, dated 1498, is made of terracotta and its size is about $70 \times 56 \times 30 \mathrm{~cm}$.

The sculpture is very rich in detail, particularly on the armor where you can see an eagle with spread wings holding in its beak a ring and leans on armors. On the shoulder of the right there is the caduceus, the symbol of reason and peace and on the left a warrior who holds the temple of Janus, with the doors opened, remembers the war against Charles VIII.

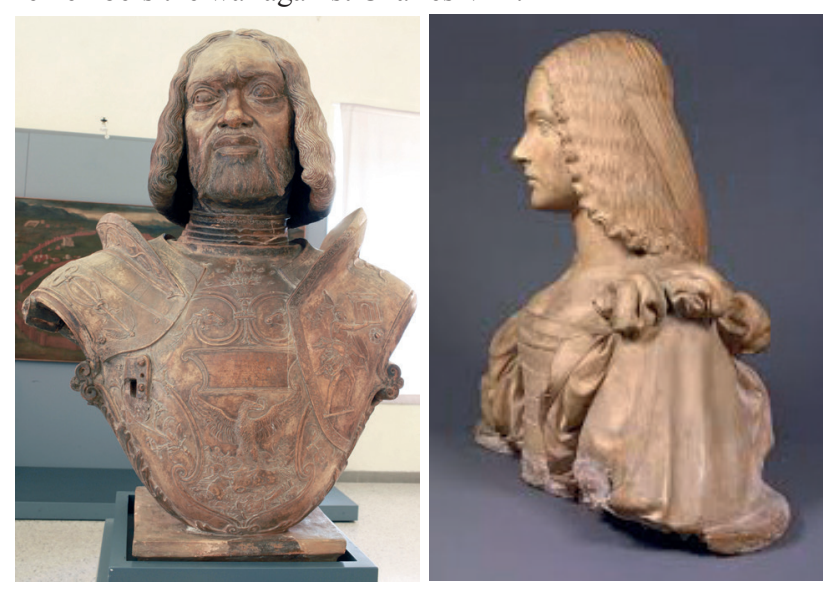

Figure 1: on the left, the bust of Francesco II Gonzaga in the museum of Mantova, on the right the bust of Isabella d'Este of the Kimbell Art Museum in Texas (USA). Both sculptures are the work of Gian Cristoforo Romano

The bust of Francesco II Gonzaga was considered until the nineteenth century work of Andrea Mantegna to the similarity with another portrait of the Marquis of Mantua made from the same Mantegna. Only in 1888 it was attributed, to tell the truth in a way not fully shared, to the sculptor Gian Cristoforo Romano who also built other works for Isabella d'Este as the design of the door of her dressing room or a famous medal exposed to Kunsthistotisches Museum in Vienna (Signorini, 2006).

The bust shows elements in common with the one made by the same Gian Cristoforo Romano for Beatrice d'Este in Milan, now preserved at the Louvre in Paris. And also shows even more in common with the bust of Isabella d'Este of The Kimbell Art Museum in Fort Worth (Texas, USA). Although the two busts are very similar and portray people very close together (husband and wife), they have never been seen in the same exposure (figure 1).

\section{3D ACQUISITION OF THE BUST}

\subsection{Methods and instruments}

Today digital technologies offer a variety of methods for the digitization of cultural heritage. All methods are typically based on optical systems that enable the 3D digitization of the object. In the field of optical sensors, two major families of sensors can generally be distinguished: active and passive ones. The difference between these two groups is based on the emission of an electromagnetic signal. Passive sensors, which are used in photogrammetric applications, simply use the ambient light to make measurements. Active sensors instead, used in laser scanner (TOF) and range cameras (based on the triangulation principle) require emitted signals and read the reflected answer. Both of these systems are applied in the $\mathrm{CH}$ as they are contactless measurement techniques and therefore they are not risky for cultural assets. Also the radiation emitted by the active sensors is not dangerous for cultural heritage (Guidi ed al, 2010).

It is not so easy to identify the most appropriate technology for 3D digitalization because there are several aspects to consider to make a choice. You must consider the characteristics of the object (shape, color, reflectivity, homogeneity of the material, etc.), the place for the acquisition (in laboratory or at the museum, with natural or artificial light, with the possibility to move the object or not), the ultimate goal (documentation, virtual reality applications, real time applications) and the economic availability.

Please refer to the large literature to examine these issues (Guidi, Remondino, 2012; Lingua et al, 2003; Beraldin, 2004), while in this article the focus regards two methods for data acquisition.

The first technique involves the use of active sensors. Range camera are based on the triangulation principle which allows to detect the position of a point in 3D space from two known points: the signal emitter and the sensor. There are many technologies which differ for the type of signal emitted: single spot, linear, fringes of light.

At the City Museum of Mantua two Minolta instruments were employed: Vivid 9i and Range 7.

They are both triangulation system with projection of a laser light blade. The difference between the two systems is given in terms of resolution and accuracy that the two instruments can reach as shown in Table 1. These instruments allow you to change their resolution by using different lenses and, consequently, also the acquisition box changes: the greater the acquisition box, the lower the resolution. As concerning colors, only the Vivid 9i has the ability to record RGB values (although not high quality), while the range 7 , born for industrial applications, can acquire only the geometric data.

These instruments need light conditions which allow to see the laser footprint on the object (they work very well in the dark too) and they are influenced, in part, by the characteristics of the surface (not suitable for reflective or transparent objects). They are suitable for objects that are not too large (approximately $1 \mathrm{~m}$ x $1 \mathrm{~m}$ x $1 \mathrm{~m}$ ).

The second approach is related to digital photogrammetry and it is based on dense image matching. They take advantage of the well-known SIFT and SURF algorithms that are able to to identify high number of key points on the images and find correspondences between stereoscopic pairs in a fast and reliable way. This let an automatic bundle adjustment and a real time 
selfcalibration. The last steps regards the construction of the mesh and the image mapping.

\begin{tabular}{|c|c|c|c|}
\hline $\begin{array}{l}\text { Range } \\
\text { camera }\end{array}$ & $\begin{array}{l}\text { Scan range } \\
{[\mathrm{mm}]}\end{array}$ & $\begin{array}{l}\text { Accuracy } \\
{[\mathrm{mm}]}\end{array}$ & $\begin{array}{l}\text { Maximum } \\
\text { Acquisition box } \\
{[\mathrm{mm}]}\end{array}$ \\
\hline \multicolumn{4}{|l|}{ Vivid 9i } \\
\hline & $\begin{array}{l}600-1000 \\
500-2500^{*} \\
(* \text { extended } \\
\text { mode })\end{array}$ & $\begin{array}{l} \pm 0.008 \\
\text { using Tele }\end{array}$ & $\begin{array}{l}\text { Tele } 463 \times 347 \times 680^{*} \\
\text { Med } 823 \times 618 \times 1100 \\
\text { Wide } 1495 \times 1121 \times 1750 *\end{array}$ \\
\hline \multirow[t]{2}{*}{ Range 7} & $450-800$ & \pm 0.004 & Tele $141 \times 176 \times 97$ \\
\hline & & & Med 267×334x194 \\
\hline
\end{tabular}

Table 1: comparison in terms of accuracy, range, acquisition box

These applications are spreading very quickly because they are easy to use and they do not require great expenses in terms of instruments and software. However it is necessary to follow well-defined procedures both in the phase of image acquisition either in data processing phase. They also require the same expedient in data modelization, derived from topographic and photogrammetric disciplines (Remondino et al, 2014).

The method is light dependent as it is based on photographs and also it requires to pay close attention to the photographic acquisition phase. As the algorithms search automatically for matching points, the surface of the objects has to be not reflective or transparent and not homogeneous.

\subsection{The acquisition stage}

The acquisition of the bust of Francesco II Gonzaga was carried out in two days. The bust was placed in the middle of the room to allow the acquisition all around. A particular illumination was not set up as the light in the room was quite diffuse.

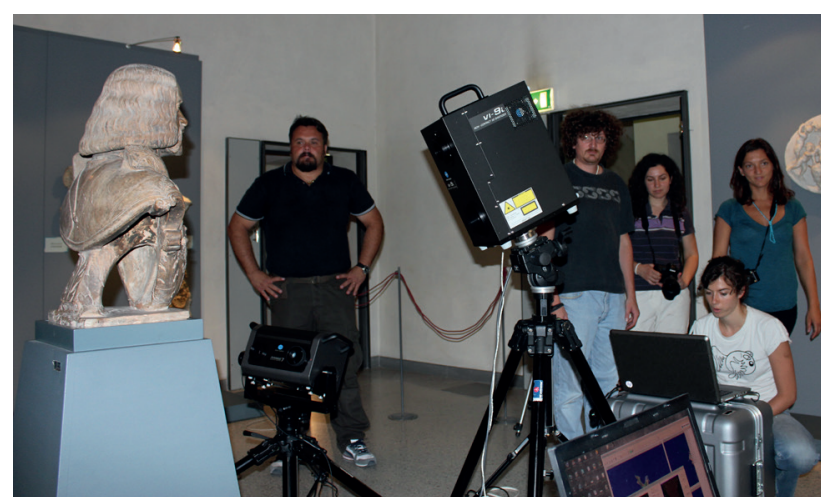

Figure 2: image of the acquisition stage with two range cameras: Minolta Range 7 (centre) and Minolta Vivid 9i (right)

\subsubsection{Acquisition by triangulation scanner}

The range camera acquisition was done using two different instruments: the Minolta Vivid 9i and Minolta Range 7 (Figure 2).

The acquisition by Minolta Vivid 9i (Minolta Vivid 9i) with medium lens, was made from a distance of about $150 \mathrm{~cm}$ from the object, with an acquisition box of about $0.55 \times 0.45 \times 0.2 \mathrm{~m}$ and an average resolution of about $0.878 \mathrm{~mm}$. The instrument Vivid 9i allows to capture also the RGB value, using a color filter in front of the lens, but the quality is quite low as influenced by ambient light. The spatial resolution of this instrument was not very high and, even if it allows to acquire also the radiometric value, we supposed that was not suitable for the documentation of the bust.

So we decided to proceed with a second instrument, Minolta Range 7, that has higher resolution even if it does not allow to acquire the radiometric value.

The work was divided into two parts.

The aim of the first stage was to detect the entire object. So the bust was acquired from different points of view, paying attention to detect all undercuts. In this case it was chosen to use the medium lens that allows a resolution of $0.212 \mathrm{~mm}$ at a distance of $70 \mathrm{~cm}$.

To detect the entire sculpture 115 scans were made. It was necessary to align scans directly in the museum through a first

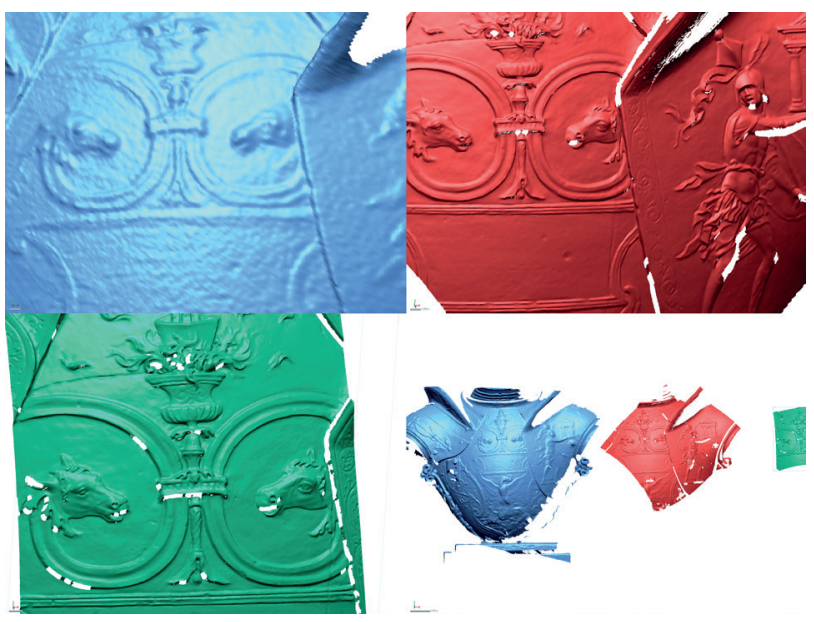

Figure 3: comparison between three scans of the same detail.

rough alignment with the software Range Viewer, in order to check the coverage of the entire statue. However some parts were not acquired as they were in hidden areas (inner surface of the trunk) or in areas where the light signal was not taken up by the sensor (occlusion of the triangle sensor-transmitter-point) .

The second phase of the acquisition regarded the acquisition of only a part of the bust, but using the maximum resolution of about $0.121 \mathrm{~mm}$. So, using the Tele lens, we scanned the area where, according to historians, there should have been some engraved words. In this second phase 20 scans were made only for the front of the armor.

In Figure 3 we can observe the comparison between scans made with the Minolta Vivid 9i with medium lens (blue), the Range 7 with medium lens (red) and the Range 7 with Tele (green) at the same distance. From the image it is evident that a smaller captured area corresponds to a higher resolution.

\subsubsection{Acquisition by photos}

The acquisition was made by using the digital camera Canon Eos 5D Mark II with $35 \mathrm{~mm}$ lens. The images are 5616 by 3744 pixels.

The photos were made moving all around the sculpture. This geometric scheme provided 30 photos taken from all around the statue and it was repeated at four different heights to cover the undercuts. In this way we can guarantee a good overlapping between images to allow SIFT algorithm to find points automatically.

The average distance from the object was about $80 \mathrm{~cm}$ and 128 images were taken. It is thus obtained a resolution of about 0.14 
mm per pixel.

The images were acquired without using a specific light source, but taking advantage of the ambient light widespread. This was made possible by the fact that the surface in terracotta is opaque and therefore it does not present particular glare or reflections.

\subsection{Data processing}

The three datasets were processed individually both for the alignment step and for the cleaning step. In particular, the scans were already in scale, each in its own reference system, while the photogrammetric model was out of scale. Then the different data sets were georeferenced in the same reference system using 6 points accurately collected on high resolution scan and used as GCP (Ground Control Points) for the photogrammetric process, to allow a metrical comparison in addition to quality one. In the last applications of digital photogrammetry the results are very similar to laser scanner ones, so after a different initial procedure, the data processing stage can be considered the same for each method.

\subsubsection{Elaboration of the scans}
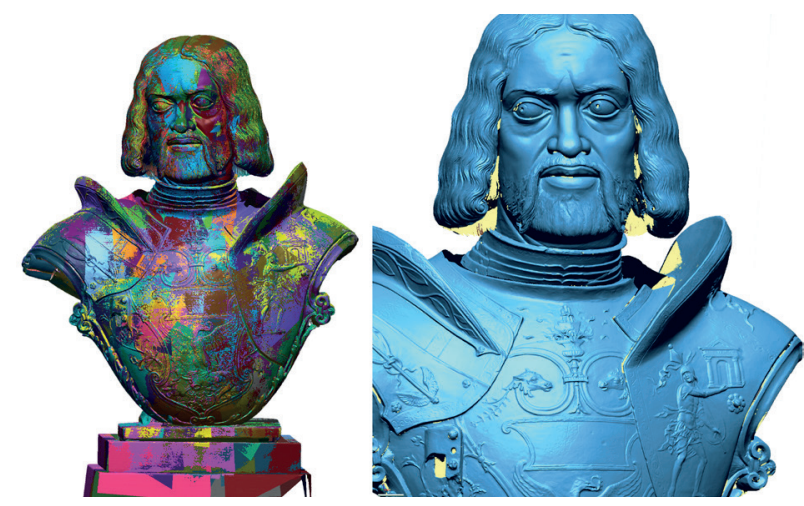

Figure 4: the model 2 made by each single scan and then merged in a single mesh. The missing part in yellow are evident and they are concentrated in the area under the head of hair.

By range camera three different data sets were acquired: Model n. 1 acquired by Minolta Vivid with Medium lens; Model n.2 acquired by Minolta Range 7 with Medium lens; Model n. 3 acquired by Minolta Range 7 with Tele lens.

The used elaboration pipeline is quite standard:

i) a first rough alignment made directly to the museum;

ii) refining of the registration with a global alignment. The residual of each alignment is equal to about 1.5 times the resolution of the scan. In the case of the dataset 2, for example, the standard deviation is approximately $0.035 \mathrm{~mm}$;

iii) filtering to remove any edge effects or small noise;

iv) merging of the scan in a single file and delete the redundant part;

v) filtering and decimation;

vi) re-meshing;

vii) filling holes.

The operations of the closure of the holes were made only for the smaller holes, while the larger gaps were left because it was quite impossible to reconstruct these parts.

At the end of data processing, the model n.,1 realized with the Minolta Vivid 9i, is composed by more than 9 million faces, but its resolution is not sufficient to accurately grasp the details. The radiometric value acquired with the scanner is not enough to

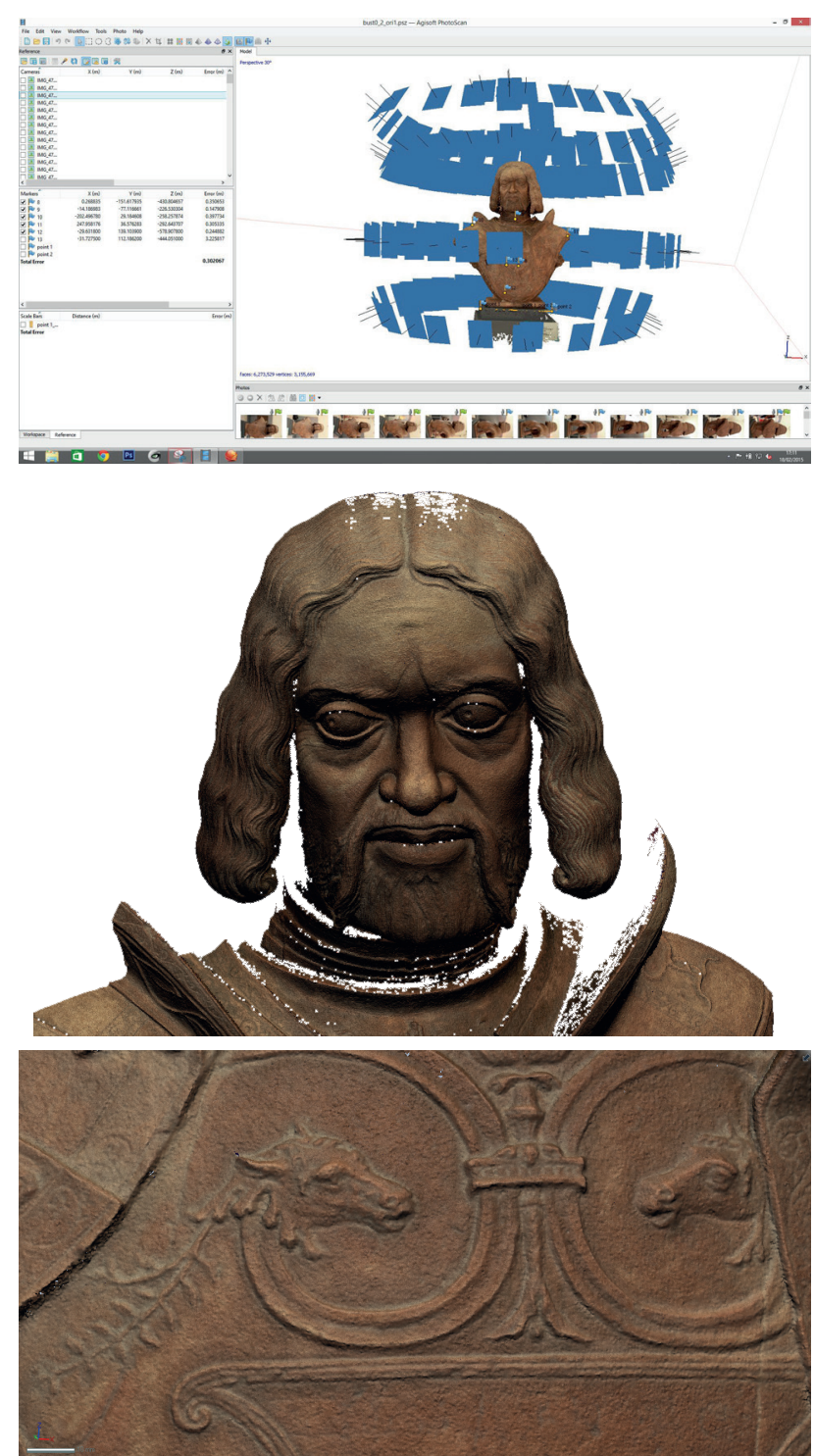

Figure 5: a) image orientation and 3D model of the bust b) detail of head and the armor

compensate for the low spatial resolution.

The second model is made up of over 38 million polygons and has no color information. The geometric resolution, however, is enough to allow to understand even the smallest details (Figure 4). The model 3 is composed of about 3.5 million triangles. But it is only a partial model because there was no attempt, in this case, to cover the entire bust. For time limitations only a test was done to achieve maximum detail with range cameras.

\subsubsection{Image based elaboration}

Photographic data followed the standard procedure of dense stereo matching software.

In Agisoft Photoscan images have been oriented automatically. Subsequently, after an initial cleaning of outliers, the process was optimized to calculate the correct calibration of the digital camera.

The obtained model was scaled and geo-referenced through a roto-translation scale variation and the coordinates were extracted directly from the model 2 . The residual on the georeferencing of was $0.3 \mathrm{~mm}$.

The dense point clouds was calculated using Sure which allowed to build a full resolution a point cloud. In Sure (Rothermel et al, 
2012) it is possible to use the orientation calculated in exterior software and to better define the characteristics of the point cloud (block analysis, dense cloud generation, point cloud filtering). The images were processed at the highest resolution, without any resample of pixels. At the end the point cloud model was made of 56 million points and this operation took about 9 hours.
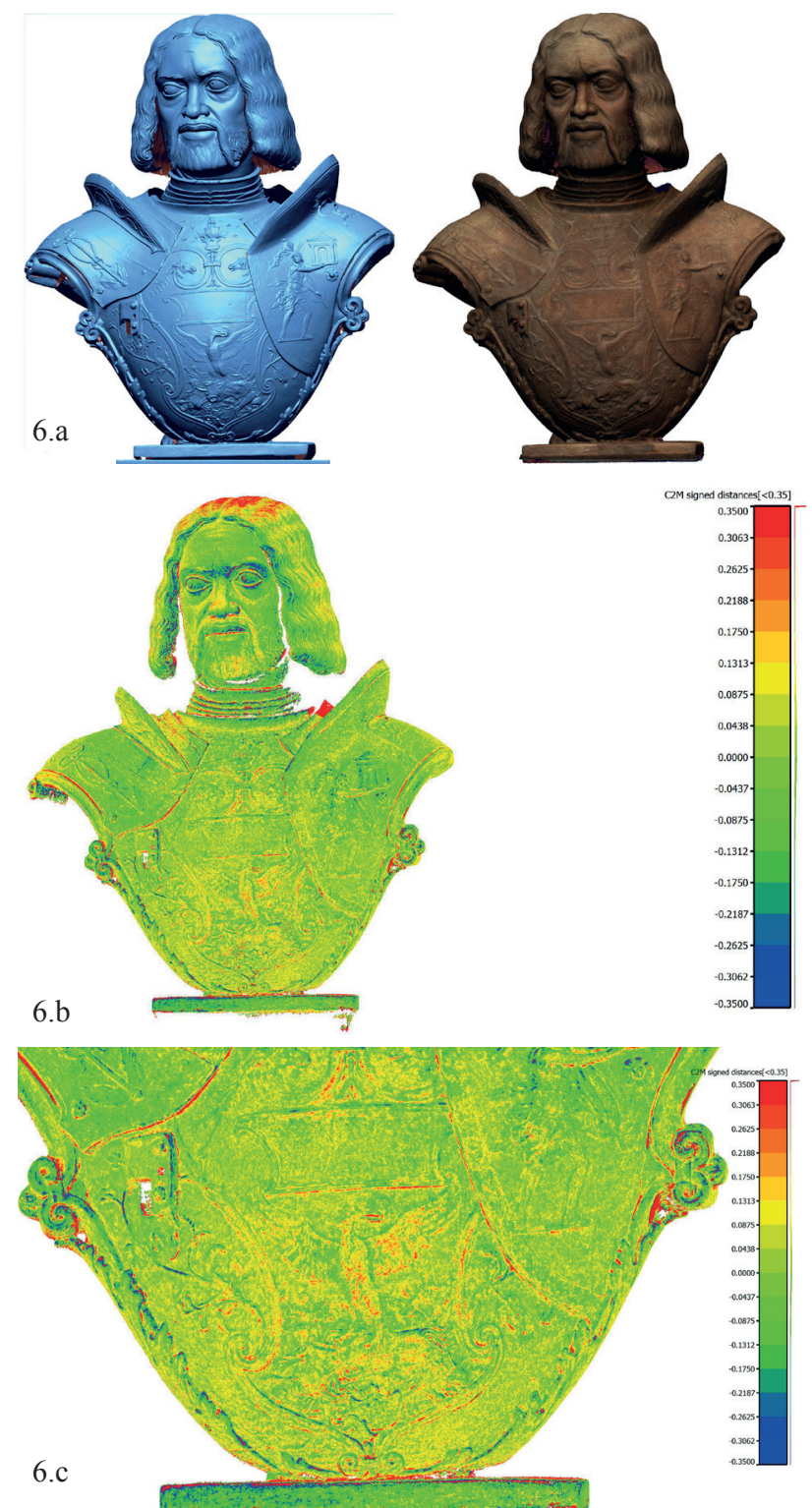

Figure 6: a) the two models compared: on the left the range camera model, on the right the photogrammetric one;

b)comparison between two models: the maximum positive and negative difference is $0.35 \mathrm{~mm}$ where there are no holes.

c) detail of the comparison of a part of the armor

The process to obtain the final mesh was quite complex because of the big size of data and the noise of the point cloud. As concerning this last point it is necessary to underline that the noise is the result of the highest resolution processing and it is strictly related to the photographic quality of images.

So data processing had to face the problem of filtering points: find a correct level of smoothing in order to preserve the finest details and delete noisy points at the same time.

To arrive to a usable model, it was necessary to split the bust into two parts: front and back, each one made of more than 53 million points.

Also in this model it can be noticed that some parts of the bust are lacking. The reasons are those already observed and linked to the inaccessibility of some parts of the sculpture. In some other cases, the lack of data is due to the fact that the surface to be restituted does not appear at least in two images (such as hair).

\section{COMPARISON OF ACQUIRED RESULTS}

At the end of data processing we obtained models with different resolutions and precision: some of them are characterized by a texture, some others have only a geometric content.

Obviously each model is particularly suitable for specific uses. The model n. 2, made by range camera, is certainly already a good starting point for the construction of a digital copy. The model built by photogrammetry, with texture, is not only for print but also for display

Also the model n. 3 is very detailed, but it is incomplete and therefore it can not be used to construct the general model of the object.

An attempt was therefore to compare the best results for documentation of the bust not only in qualitative, but also in quantitative terms.

The model obtained by photogrammetry was compared with the model n. 2 obtained through the Minolta Range 7 with medium lens.

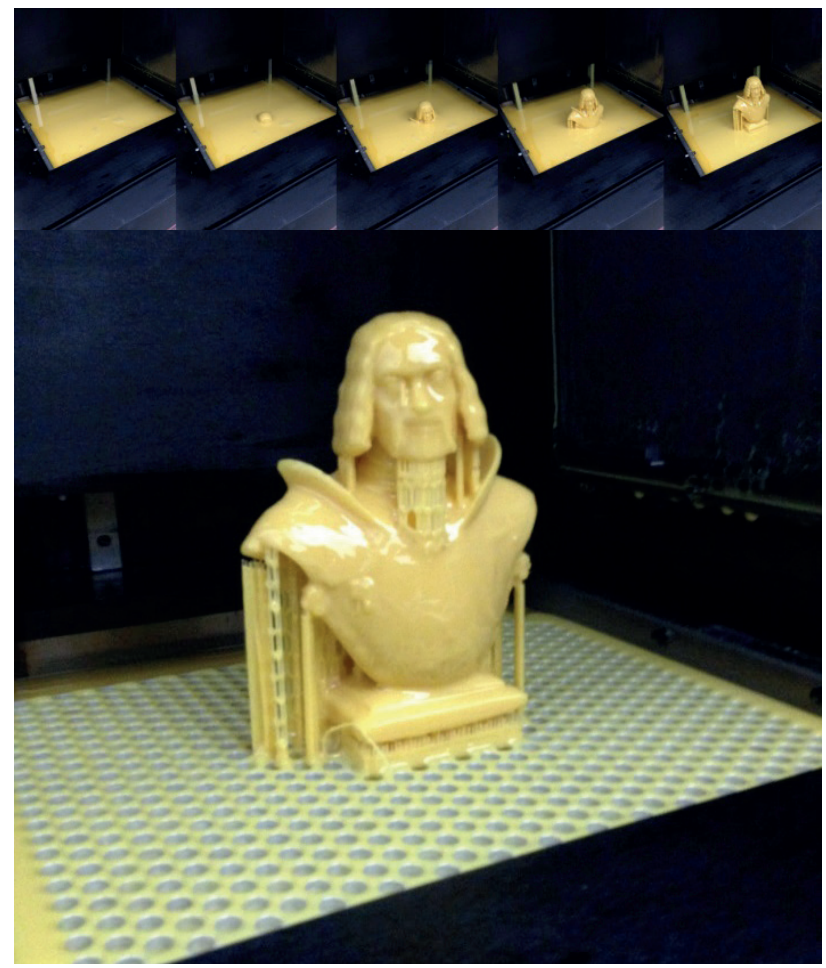

Figure 7: Above: different steps of the construction of the resin solid. Below: the 3D printed model on the plane of the printer, still with supports.

The analysis was carried out with the open source software Cloudcompare (Cloudcompare) that allows to measure the distance between two surfaces or two point clouds.

The software Cloudcompare allows also to align the models assuming also a transformation of scale. But in this case it was not necessary to apply the scale transformation as the operation of georeferencing had a very low residual. This operation is however advisable when the model is scaled with a known distance or the precision of the coordinates is not very high. 
The comparison between the two models showed that the differences between the two models are very small (the mean error is $0.0319 \mathrm{~mm}$ with a standard deviation of $0.129 \mathrm{~mm}$ ).

The main differences ara along the discontinuity where the point cloud is more noisy and with a high number of outliers (Figure $6)$.

\section{3D PRINT}

In the last years, the advent of $3 \mathrm{D}$ printing has opened new scenarios and possibilities related not only to the production of objects of common use (design), but also to the documentation and fruition of Cultural Heritage. As we can see in literature or in several web sites, the experiences of replicas of objects, which are exhibited in museums as faithful copies of the original or reconstructions or to set up museum tours for the blinds, are growing (Neumuller et al. 2014; D'agnano et al. 2015).

Contrary to $\mathrm{CNC}$ machines (Computer Numerical Control), working with subsequent subtraction of material from a block in which is "contained" the shape you want to get, the new Rapid Prototyping systems, generally 3D printer, manufacture successive layers of material (additive manufacturing). The main differences between additive processes are in the way layers are deposited (extrusion, photo-polymerization, lamination, etc.) to create parts and in the materials that are used (liquids, powders, filaments, laminated, etc.). Layer after layer, these printers reconstruct the object representing the mathematical model realized by the point cloud surveyed.

The process for the realization of the printed copy is already well known. In this experience, the 3D model (mesh) of the bust was obtained by Range 7 data (model n. 2).

Some minor editing of the mesh were necessary only to assure the watertight less off the mesh and to prepare templates for printing (empty the model, prepare supports).

Two different 3D printer were used to compare the quality in printing and the differences in models details: a low cost one (Solidoodle) with ABS filament, and a professional one (Ultra 2 by Envisiontech) based on the technology of image projection on curing resins. These two printer have also different resolutions as evident in Table 2.

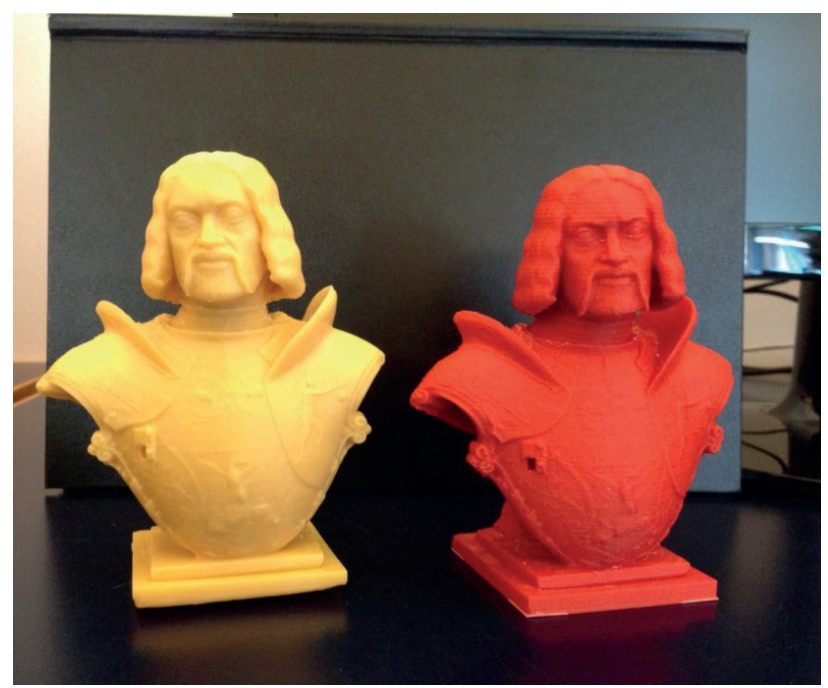

Figure 8: Resin (yellow) and ABS (red) 3D sculpture printed at scale 1:5

The STLs are optimized (sizing, placing, slicing) in the two different proprietary software managing the printing process. In both cases, the printing time (about 8 hours) depend on the height

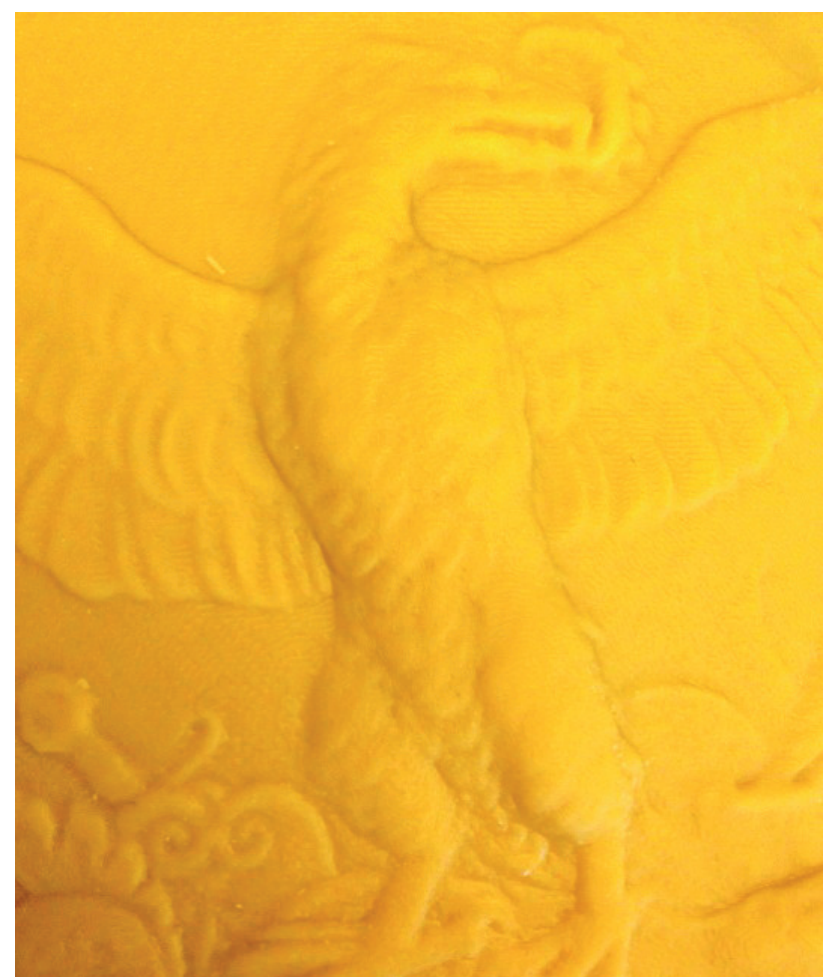

Figure 9: A detail of the armor at scale 1:1

of the object.

\begin{tabular}{|l|l|l|l|}
\hline & $\begin{array}{l}\text { XY resolution } \\
{[\mu \mathrm{m}]}\end{array}$ & $\begin{array}{l}\text { Z resolution } \\
{[\mu \mathrm{m}]}\end{array}$ & $\begin{array}{l}\text { Maximum } \\
\text { printable area } \\
{[\mathrm{mm}]}\end{array}$ \\
\hline Solidoodle & 11 & 100 & $154 \times 154 \times 154$ \\
\hline Ultra 2 & 50 & $15-100$ & $267 \times 165 \times 203$ \\
\hline
\end{tabular}

Table 2: comparison between two solid printers

After printing following steps are needed: elimination of the supports, any finish of paper with slightly abrasive, fixing resin. The model of the whole bust was printed in a reduction scale 1:5 according to its resolution (Figure 8).

The two prints are clearly different as related to different resolutions and characteristics of printers. The quality is sufficient, however, to allow their use in advertising or marketing operations for cultural heritage. At a finest analysis, in the model printed by Solidoodle the layers of materials are recognizable, instead the other print is more homogeneous.

Morover, a detail of the armor was printed with the 3D professional printer by resin to use the maximum achievable resolution. We used the model n. 3, the most detailed one, and we printed in a scale 1:1. (Figure 9).

Finally all these solid models can be finished with ad hoc finishes to simulate the original material or to bring out the finer details.

\section{CONCLUSIONS}

Geomatics techniques and methods are now able to provide a great contribution to Cultural Heritage, being adaptable to different purposes: management, diagnosis, restoration, protection, study and research, communication, formation and fruition of the Cultural Heritage. This research, concerning the survey of the bust of Francesco Gonzaga II, aimed to obtain a 
high fidelity digital copy to document its state of conservations and to demonstrate the importance of 3D printed replica.

The models obtained by photogrammetric technique or by triangulation scanners present very small geometric differences. Nowadays triangulation scanners and photogrammetry can reach the same resolution, necessary to a $3 \mathrm{D}$ print at scale $1: 1$, which can be assessed, in our opinion in 02 microns. The opportunity to choose one method or another are no more related to the final resolution and accuracy, but to some "external conditions": the economic resources, the availability of powerful pc, the ability of acquiring high resolution and really perfectly focused images. Range camera require a greater financial commitment, but data processing is quite fast and automatic, partly because the result of the scan is a structured triangulated mesh. Photogrammetry, however, against lower costs, requires more attention to the step of image acquisition (considering the problems of lighting, focus, depth of field) and requires more computing power for the phases of orientation and calculation of the dense cloud.

Added value of the photogrammetric process is the possibility to have at disposal a metrically reprojected high resolution and quality texture. The choice depends also on the final application: for a solid print the geometry is fundamental, for visualization and diagnoses, the texture is unavoidable.

Regarding 3D printing, as can be understood from this experience, it represents a new important tools not only in the field of reconstruction of objects (as archaeology), but also for research, documentation, and educational purposes.

The re-contextualization of the asset respect the original project of the artist or the historical and cultural background, it's another important aspect: in the case of the busts made by Giancristoforo Romano, this could permit to position the Francesco II Gonzaga statue and the copy of the bust of Isabella Gonzaga together in the same place.

The $3 \mathrm{D}$ print has the potential to serve all these purposes in a very accessible way.

\section{ACKNOWLEDGEMENTS}

Many thanks to dr. Stefano Benetti and dr. Chiara Pisani of the City Museum of Mantua for giving permission for the digitization of the bust and to have facilitated the acquisition.

\section{REFERENCES}

Adami A., Carlani R., Van Kampen I., Pietroni E., and Sannibale M. 2012. Digital techniques for Etruscan graves: The etruscanning project. In Proceedings of VAST 2012;

Agisoft, http://www.agisoft.com/, last visit on 28 February 2015;

Balletti C., Adami A., Guerra F., Paolo Vernier P., 2012, Dal rilievo alla maquette: il caso di San Michele in Isola, in Archeomatica, year 3, number 2;

Beraldin A., 2004. Integration of laser scanning and closerange photogrammetry-The last decade and beyond. In The International Archives of the Photogrammetry, Remote Sensing and Spatial Information Science Congresss. Comission VII, Istanbul, Turkey;

Cloudcompare, http://www.danielgm.net/cc/, last visit on 28 february 2015 ;

D’Agnano F., Balletti C., Guerra F., and Vernier P., 2015. TOOTEKO: a case study of augmented reality for an accesible cultural heritage. Digitization, 3D printing and sensors for an audio-tactile experience, Int. Arch. Photogramm. Remote Sens. Spatial Inf. Sci., XL-5/W4, 207-213, doi:10.5194/isprsarchivesXL-5-W4-207-2015, 2015;

FASSI F., FREGONESE L. ACKERMANN S., DE TROIA V., (2013) Comparison between laser scanning and automated $3 \mathrm{D}$ modelling techniques to reconstruct complex and extensive cultural heritage areas. ISPRS Workshop 3D-ARCH: 3D virtual reconstruction and visualization of complex architectures, Trento, 2526 February 2013

Guidi G., Remondino F. 3D modeling from real data, 2012 In Modeling and Simulation in Engineering, Alexandru C. Ed., InTech: Rijeka, Croatia, 2012, pp. 69 - 102;

Guidi G., Russo M., Beraldin J.A., 2010, Acquisizione 3D e modellazione poligonale, McGraw-Hill, Milano;

Lingua A., Piumatti P., Rinaudo F. , 2003,Digital photogrammetry: a standard approach to cultural heritage survey. In proceedings of International Archives of the Photogrammetry, Remote Sensing and Spatial Information Sciences, Ancona, Italy, July 1-3, 2003, Vol. 34-5/W12, pp. 210-215;

Minolta Range 7, http://www.konicaminolta.it/measuringinstruments/products/for-3D-measurement/non-contact-3Ddigitizer/konica-minolta-range $7 . h t m l$, last visit on 28 February 2015;

Minolta Vivid 9i, http://www.konicaminolta.it/measuringinstruments/products/for-3D-measurement/non-contact-3Ddigitizer/vi-9i.html, last visit on 28 February 2015;

Neumuller M., Reichinger A., Rist F., Kern C., 2014, 3D Printing for Cultural Heritage: Preservation, Accessibility, Research and Education. Lecture notes in Computer Science. Springer, 8355; 119-134. doi: 10.1007/978-3-662-44630-0_9;

Remondino, F., Spera, M. G., Nocerino, E., Menna, F. and Nex, F. (2014), State of the art in high density image matching. The Photogrammetric Record, 29:144-166. doi10.1111/phor.12063;

Rothermel, M., Wenzel, K., Fritsch, D., Haala, N. 2012, SURE: Photogrammetric Surface Reconstruction from Imagery. Proceedings LC3D Workshop, Berlin ,December 2012;

Signorini R. edited by, 2006, A casa di Andrea Mantegna. Cultura artistica a Mantova nel Quattrocento, Catalogo della Mostra, ed. Silvana editoriale, Milano;

Solidoodle, http://www.solidoodle.com/, last visit on 28 February 2015;

Taffurelli L, Fregonese L., 2011, Transmission of cultural heritage through time and space by digital 3D models, Proceedings XXIII CIPA Symposium - Prague, Czech Republic;

Ultra2 by Envisiontec, http://envisiontec.com/, last visit on 28 February 2015. 